\title{
Management of depressive disorder in adolescents
}

\author{
Richard Harrington
}

Historically, depressive disorders in adolescents have been conceptualised as variants of the nonspecific class of childhood emotional or neurotic disorder, which encompassed depressive, anxiety, hysterical and obsessional disorders. Over the past decade, however, the field has shifted towards greater diagnostic refinement. Research evidence has shown that severe depressive disorders differ from other mental disorders in this age group in their epidemiology, outcome and correlates (Harrington \& Wood, 1995). Treatment therefore includes not only general techniques of clinical management, but also a variety of treatments targeted at the symptoms of depressive disorder.

\section{Diagnosis and assessment}

Defining the boundaries between extremes of normal behaviour and psychopathology is a dilemma that pervades all of psychiatry. It is especially problematic to establish the limits of depressive disorder in adolescents because of the cognitive and physical changes that take place during this time. Adolescents tend to feel things particularly deeply and marked mood swings are common during the teens. It can be difficult to distinguish these intense emotional reactions from depressive disorders.

Assessment of adolescents who present with symptoms of depression must, therefore, begin with the basic question of diagnosis (Box 1). Standardised diagnostic systems such as the DSMIV (American Psychiatric Association, 1994) and structured psychiatric interviews can help in deciding whether the patient has serious symptomatology that requires treatment.
Unfortunately, these diagnostic systems tend to be overinclusive in this age group and many dysphoric adolescents who meet criteria for so-called major depression remit within a week or two. It is important, then, that careful enquiry is made about the impact that the adolescent's symptoms have had on everyday functioning and about the presence of symptoms of unequivocal psychopathological significance, such as suicidal planning or marked weight loss. Probably the best single indicator of whether or not an adolescent has a serious depressive disorder is the duration of the problem. Polysymptomatic depressive states that persist for more than six weeks usually require treatment. Severity of depression can also be assessed by depression scales such as the Mood and Feelings Questionnaire (Angold et al, 1987), which has been validated in clinical trials with depressed adolescents (Wood et al, 1995), or the Children's Depression Inventory (Kovacs, 1981). These scales are also a useful way to monitor treatment response. However, it should be borne in mind that they are not intended to be diagnostic. Most depression questionnaires have low specificity for depressive disorder, and as a result many nondepressed adolescents will have high scores on

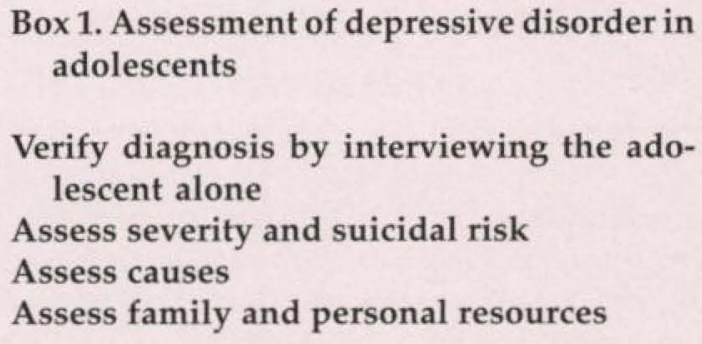

Box 1. Assessment of depressive disorder in adolescents

Verify diagnosis by interviewing the adolescent alone

Assess severity and suicidal risk

Assess causes

Assess family and personal resources

Professor Harrington is Chair of the Department of Child and Adolescent Psychiatry at the University of Manchester and a consultant at Royal Manchester Children's Hospital, Pendlebury, Manchester M27 1HA. One of his main clinical and research interests is affective disorder in children and adolescents. 
such scales. Moreover, some severely depressed adolescents have low scores.

Assessment of the depressed adolescent should also include careful enquiry about comorbid disorders, particularly anxiety and conduct disorders. In research settings it is common to diagnose depressive disorder in adolescents regardless of comorbid disorders. However, in clinical practice it is much better to reserve the diagnosis for cases in which depression is a prominent part of the clinical picture. Such cases are more likely to persist (Harrington et al, 1990).

Although the accurate diagnosis of depressive disorder is an important part of clinical management, assessment only starts with the diagnosis, it does not stop with it. Aetiology should be assessed next, with respect to precipitating, predisposing and maintaining factors. No attempt to allocate the syndrome to an exclusively endogenous or reactive category should be made. Instead the importance of both external and internal causes should be assessed in every case. Depressed adolescents usually have multiple problems such as educational failure and impaired psychosocial functioning. They find it harder to make and maintain friendships than their peers and they tend to come from families with high rates of mental illness. There is often a precipitant for the episode of depression and some depressed adolescents have been maltreated or abused. All these problems need to be identified and the causes of each assessed.

The final part of the assessment involves the evaluation of the adolescent's personal and social resources. There is evidence that being successful at school or in other areas of life can protect young people from the effects of adverse life experiences (Quinton et al, 1984). The best guide to the adolescent's ability to solve future problems is his or her record in dealing with difficulties in the past. The ability of the family to support the adolescent should also be evaluated.

\section{General principles of treatment}

Many of the general principles of treatment follow from the formulation of depressed adolescent's difficulties outlined above. Comorbidity is frequent, there are many complications, and other types of adversity are part of the cause and may need intervention in their own right. The course is determined by more than just the presence and severity of depression (Harrington \& Vostanis, 1995). Therefore, attention must be paid to
Box 2. General principles of treatment

\section{Initial treatment}

Rapid assessment

Explain nature and causes of symptoms to the adolescent and the family

Reassure that in most cases remission will occur within a few months

Give help in solving problems that might be causing the depression or in coming to terms with them

\section{Further treatment}

Individual psychological treatment for the adolescent, such as cognitive-behavioural therapy

Help for the family

If depression is severe, consider antidepressant treatment

Measures to treat concomitant psychopathology

General measures to foster normal development

\section{Follow-up}

Continuation treatment for cases at high risk of relapse

'Check-ups' for one year for the remainder

biological, familial, educational and peer contributions. As with other child psychiatric disorders, it is important not only to treat the presenting problems but also to foster normal development. A treatment programme has multiple aims: to reduce depression, to treat comorbid disorders, to promote social and emotional adjustment, to improve self-esteem, to relieve family distress and to prevent relapse (Box 2).

\section{Early management}

The first question is whether the depression is severe enough to warrant admission to hospital. Indications for admission of depressed adolescents are similar to those applicable to their adult counterparts and include severe suicidality, psychotic symptoms or refusal to eat or drink. A related question is whether the adolescent should remain at school. When the disorder is mild, school can be a valuable distraction from depressive thinking. When the disorder is more severe, symptoms such as poor concentration and motor retardation may add to feelings of hopelessness. 
The next issue is whether the depression is complicated by other disorders such as behavioural problems. If it is, then as a general rule it is best to sort out these complications before embarking on treatment for the depression. For example, if a child has a major behavioural problem then it will be necessary to ensure that appropriate measures are being taken to deal with this. Similarly, the depression-like states that are common in adolescents with anorexia nervosa usually respond much better to weight gain than they do to antidepressants.

The third issue concerns the management of the stresses that are found in many cases of major depression. The assessment may indicate that the reaction of the adolescent is appropriate for the situation in which she finds herself. In such a case, and if the depression is mild, it is sensible to direct efforts towards alleviation of these stresses. However, in the majority of cases acute stressors are just one of a number of causes of the depression. Moreover, acute life events in adolescents often arise out of chronic difficulties (such as family discord), which may be very hard to remedy. Symptomatic treatments of depression can therefore be helpful even when it is obvious that the depressive symptoms occur in the context of chronic family or social adversity that one can do little to change.

\section{Treatment of the acute episode}

The treatment of depressive disorder should be tailored to the results of the assessment. Treatment should include both specific treatments for depressive symptoms and management of associated problems.

\section{Psychological treatments for the adolescent}

In most cases of adolescent depressive disorder the first-line treatments are psychological. A very large number of treatments have been advocated for juvenile depressive disorders but only a few have been evaluated in randomised controlled designs. The most thoroughly studied of the individual psychotherapies has been cognitive-behavioural therapy (CBT). Studies of depressed adolescents have repeatedly shown that they have negative styles of thinking and difficulties with social relationships. Cognitive-behavioural programmes therefore include:

(1) 'cognitive' strategies, such as distraction techniques, to deal with negative cognitions;

(2) social problem-solving techniques, to deal with difficulties in social relationships;
(3) techniques to cope with symptoms of depression, such as sleep hygiene measures to deal with sleep problems and activity scheduling to deal with reduced activity.

A variety of slightly different CBT programmes have been developed and most of them have been published or are available as manuals (Zarb, 1992; Vostanis \& Harrington, 1994; Wilkes et al, 1994), so training of therapists who already have experience of working with adolescents can usually be accomplished in a few months.

Numerous studies have shown that in nonclinical samples of depressed adolescents CBTs are superior to remaining on a waiting list (Harrington, 1992). Cognitive-behavioural therapy has also been shown to be of value in moderately severe depressive disorders in clinical samples. Wood et al (1996, in press) found that CBT led to a significantly greater improvement than relaxation training in both depressive symptoms and global functioning. These changes were clinically significant and in many cases the improvements persisted for six months.

\section{Family interventions}

There is a strong association between depressive disorder in adolescents and mental problems in their parents, particularly depressive and anxiety disorders (Harrington, 1996). In part, this association may be a reflection of genetic influences. However, the linkages also arise from environmental processes such as exposure to parental criticism or from other problems in parenting. Three types of family work have therefore been used to help the families of depressed adolescents. First, families of depressed young people will often benefit from advice and counselling about depression and its associated symptoms. For example, parents of suicidal adolescents will need guidance about the best way to reduce the risk of a suicide attempt. In addition, it is often helpful to explain to the parents what is known about the probability of recovery. The great majority of depressed young people will recover eventually. Second, parents and siblings can have an important role in helping with individual therapies such as CBT. For instance, parents can help their child to become more socially active or in scheduling activities. Third, in some cases one or all members of the family may become the focus of treatment. Thus, where a parent has an overt psychiatric disorder it will be necessary to help the parent to get treatment from a mental health professional. In some families it may be necessary to see all the family members together. There is some evidence that families become 'locked' into a cycle whereby the parents' problems 
lead to depression in the child, which in turn leads to further parental problems (Hammen, 1991). Such cycles may be amenable to family therapy, though this has not yet been evaluated in controlled trials.

\section{Tricyclic antidepressant medication (Box 3)}

Medication has been more extensively evaluated in clinical depressive disorders than psychological treatments. There were promising results from early open trials with tricyclic antidepressants (TCAs), but subsequent controlled trials have all failed to show significant benefits over placebo (Harrington, 1992). It is unclear why tricyclics are less effective for depressive disorders in adolescents than they are for the apparently identical condition in adults. It has been suggested that there are developmental variations in the rate of metabolism of psychotropics, which make it harder to get the dosage right in adolescents than in adults (Ryan, 1990). It is also possible that adolescent depression, though superficially similar to adult depression, differs in some important respect that might affect drug responsiveness. For example, it could be that the cases admitted to drug trials have been more severe. Alternatively, it may be that adolescents differ from adults in the neurotransmitter systems on which antidepressants are thought to act.

These uncertainties about the efficacy of tricyclics are compounded by the fact that their administration to depressed adolescents can be quite difficult (Box 4). Many depressed adolescents are suicidal and TCAs are of course toxic in overdose. Moreover, even in therapeutic doses the TCAs are associated with changes on the electrocardiograph and, in vulnerable individuals, with untoward effects on the central nervous system, such as drowsiness or confusion. Unfortunately, in young people there are large interindividual variations in steady-state plasma levels of tricyclics, so one-off plasma levels are not a reliable guide to toxicity. It can therefore be very difficult to get the dosage right.

Box 3. Indications for antidepressant medication

Older adolescents

Clinical picture similar to adult depression Somatic symptoms

Failed to benefit from adequate course of psychological treatment

Previous positive response to antidepressants
Box 4. Baseline assessment before starting antidepressants

Psychiatric history (in particular, enquire about personal or family history of bipolar illness or psychotic disorder)

Medical history (including drug history, family history of heart disease)

Depression self-report scale (e.g. MFQ)

Full physical examination

Electrocardiograph

Full blood count, electrolytes and liver enzymes

(Pregnancy test)

It is, however, important to note that research on the effectiveness of TCAs in this age-group is necessarily at an early stage. The evidence that TCAs are effective in adult depressive disorders is so strong that there is still a case for using them as second-line treatments with older adolescents who have 'typical' depressive disorders and in whom the risk of attempted suicide is low.

\section{Serotonin-selective reuptake inhibitors}

Less is known about the effectiveness and sideeffects of the newer antidepressants such as the serotonin-selective reuptake inhibitors (SSRIs). However, on the basis of adult experience, drugs such as fluoxetine and paroxetine should be less toxic to the heart and nervous system than the TCAs. In many centres they are therefore the first choice of antidepressant. Nevertheless, all cases require a detailed baseline assessment, including a full medical history and physical examination. In addition, it should be borne in mind that at the moment SSRIs are more expensive than the tricyclics and their long-term toxicity in adolescents is unknown.

The most commonly used SSRIs with adolescents are fluoxetine, which has been evaluated in a controlled trial (Simeon et al, 1990) and paroxetine. Experience is also accumulating with sertraline and fluvoxamine; the evidence from open studies is that around $60-70 \%$ of cases will show some improvement. Dosage schedules are similar to those with adults. For example, the effective daily dose of paroxetine is usually between 20 and $40 \mathrm{mg}$ for eight to 12 weeks. It is usually best to start paroxetine with a daily dose of $10 \mathrm{mg}$ and increase slowly. Side-effects of paroxetine occur in around $15 \%$ of adult cases. The most common are sedative effects such as problems in learning, stimulant 
effects such as sleep problems, headaches and gastrointestinal disturbances (Edwards, 1994). Sexual problems such as premature ejaculation have also been reported. More rarely, some patients complain of feelings of depersonalisation and memory problems. Despite earlier concerns, there is little evidence of increased suicidality in patients taking SSRIs. It is not known whether the SSRIs are teratogenic and they should be avoided during pregnancy. The so-called 'serotonin syndrome', in which there can be severe gastrointestinal and central nervous system symptoms, is potentially a problem.

\section{Treatment resistance}

Around one-half of depressed adolescents who are referred for psychological treatment will remit within 10 weeks or so. About a half of the remainder will remit within the following three months either with SSRIs or with other treatments. However, about $20 \%$ of adolescents with severe depression who are referred to mental health services will still be depressed after six months and even after a year some $10 \%$ of cases are resistant to treatment (Harrington \& Vostanis, 1995).

If an adolescent is resistant to the effects of treatment then the reasons for this should be reviewed. It may be that other problems besides depression are present. Common causes of treatment resistance include undiagnosed psychosocial stressors such as abuse, comorbid disorders such as conduct disorder, chronic family difficulties and emerging personality problems. Less common but important causes are undiagnosed physical disorders such as endocrine disturbances (rare) and drug abuse.

Review may also indicate some specific reasons for the failure of treatment. For example, CBTs are less effective when given by inexperienced therapists who tell the adolescent what to do rather than giving the patient the opportunity to work it out on his or her own. Drugs may have been prescribed in doses that were too low or for too short a period. Occasionally, side-effects of medication such as confusional states or the serotonin syndrome may be mistaken for depression, with the paradoxical result that as drug dosage increases the 'depression' gets worse. Medication is generally less effective when depression is one pole of a bipolar disorder. There is no reliable way of distinguishing between the depressive phase of bipolar disorder and simple unipolar depression. However, a family history of
Box 5. Treatment choices for treatmentresistant depression in adolescents

Maintain ongoing treatment at current level and wait for another four weeks

Major environmental manipulation such as admission to hospital

Stop SSRI, start tricyclic

Augment tricyclic with lithium

Other psychological or social interventions

Supportive care pending remission

ECT

manic disorder or a personal history of previous manic episodes (which may be hard to recognise because the symptoms can closely resemble hyperkinetic disorder) may indicate the true nature of the underlying problem.

If the review does not show a simple way of improving the adolescent's mental state, then a different line in treatment should be pursued (Box 5). For example, it may be necessary to switch from an SSRI to a TCA or a monoamine oxidase inhibitor (though it should be remembered that SSRIs and TCAs can interact, and SSRIs such as fluoxetine have metabolites with a very long half-life). Tricyclics may be augmented with lithium. Other psychological treatments may also be indicated, such as family therapy or longer-term forms of individual therapy such as interpersonal psychotherapy. Alternatively, it may be necessary to admit the adolescent to hospital. Admission can be a valuable way of conducting an in-depth assessment and many depressed adolescents will improve in the milieu of a well run adolescent unit. Electroconvulsive therapy (ECT) is still used to treat severe affective disorders in older adolescents and there are anecdotal accounts of its efficacy in this agegroup (Moise \& Petrides, 1996). However, little is known about the long-term side-effects of ECT given to adolescents and therefore it should be used only in the most exceptional circumstances.

\section{Prevention of relapse}

Many studies have shown that young people who remit from major depression have a high risk of relapse. The risk of relapse varies from study to study, but in clinical samples is probably as high as $50 \%$ within the nine months after remission. The 
risk of relapse seems to be particularly great within a few months of remission. For example, we have found that more than $40 \%$ of adolescent patients who had responded to CBT had a relapse within three months of remission (Wood et al, 1996, in press).

A similarly high rate of relapse after remission from the acute episode has been found in adult depressed cases. However, controlled studies with depressed adults suggest that this relapse risk can be reduced by continuing either pharmacological or psychological treatments for several months after apparent remission (Kupfer, 1992). The theory underlying such continuation treatments is that suppression of symptoms by the acute phase of treatment does not necessarily indicate that the individual has completely recovered from the episode. The patient may therefore remain vulnerable to a recurrence of symptoms for several months. Continuation of treatment aims to prevent symptoms returning.

Research on the prevention of depression in young people is at an early stage. Preliminary findings from community studies of children and adolescents designated as at risk because of depressive symptoms have been encouraging to the extent that they have shown that CBT is associated

Box 6. Causes of depressive disorder in adolescents

Predisposing factors

Inherited liability to major affective disorder Inherited temperamental features that predispose to depression directly (e.g. poor emotional regulation) or indirectly (e.g. difficult temperament leading to adversity)

Early adversity and attachment problems

Negative cognitive style

Chronic difficulties, particularly within the family

Precipitants

Acute life events

Physical illness (rare)

Bereavement

Failed social relationships

School failure or bullying

Maintaining factors

Ongoing difficulties, especially within the family

Ongoing social relationship problems

Negative cognitive style with a reduced risk of subsequent depression. Continuation CBT is feasible in clinical samples and seem to be associated with a reduced risk of recurrence (Kroll et al, 1996, in press). However, it remains to be seen whether efficacy will be demonstrated in controlled trials. In the meantime, it seems sensible to continue the treatments that have led to remission for several months. Alternatively, cases at low risk for relapse should be seen regularly for 'check-ups' during which the adolescent's mental state should be assessed. Depressive disorders in adolescents require longterm approaches to treatment.

\section{Conclusions}

Over the past decade great progress has been made in the diagnosis, assessment and treatment of depressive disorders in young people. Although most adolescents with major depression will recover within a year or so, they have a high chance of another episode. It is therefore likely that future research on treatment will focus much more on the prevention of recurrence. The future also looks promising for the development of new treatments for the acute episode, such as interpersonal psychotherapy. Many of the older treatments, such as the SSRIs and CBT, require further controlled evaluation in clinical samples. The knowledge gained from the new generation of controlled studies will eventually be of great benefit to depressed adolescents and their families.

\section{Acknowledgements}

This work was made possible by grants from the MacArthur Foundation Network on Psychopathology and Development, the Medical Research Council and the Mental Health Foundation.

\section{References}

American Psychiatric Association (1994) Diagnostic and Statistical Manual of Mental Disorders (4th edn) (DSM-IV). Washington, DC: APA.

Angold, A., Costello, E. J., Pickles, A., et al (1987) The Development of a Questionnaire for use in Epidemiological Studies of Depression in Children and Adolescents. London: Medical Research Council Child Psychiatry Unit.

Edwards, J. G. (1994) Drugs in focus: 14. The selective serotonin reuptake inhibitors in the treatment of depression. Prescribers' Journal, 35, 197-204.

Hammen, C. (1991) Depression Runs in Families. The Social Context of Risk and Resilience in Children of Depressed Mothers. New York: Springer-Verlag. 
Harrington, R. C. (1992) Annotation: the natural history and treatment of child and adolescent affective disorders. Journal of Child Psychology and Psychiatry, 33, 1287-1302.

- (1996) Family-genetic findings in child and adolescent depressive disorders. International Review of Psychiatry, in press.

, Fudge, H., Rutter, M., et al (1990) Adult outcomes of childhood and adolescent depression: I. Psychiatric status Archives of General Psychiatry, 47, 465-473.

—\& Vostanis, P. (1995) Longitudinal perspectives and affective disorder in children and adolescents. In The Depressed Child and Adolescent. Developmental and Clinical Perspectives (ed. I. M. Goodyer), pp. 311-341. Cambridge: Cambridge University Press.

— \& Wood, A. J. (1995) Validity and classification of child and adolescent depressive disorders. Review of the field circa 1995. In Childhood Depression. ACPP Occasional paper No. 11 (ed. G. Forrest), pp. 3-22. London: ACPP.

Kovacs, M. (1981) Rating scales to assess depression in school aged children. Acta Paedopsychiatrica, 46, 305-315.

Kroll, L., Harrington, R.C., Gowers, S., et al (1996) Continuation of cognitive-behavioural treatment in adolescent patients who have remitted from major depression. Feasibility and comparison with historical controls. Journal of the American Academy of Child and Adolescent Psychiatry, in press.

Kupfer, D. (1992) Maintenance treatment in recurrent depression: current and future directions. British Journal of Psychiatry, 161, 309-316.

Moise, F. N. \& Petrides, G. (1996) Case study: electroconvulsive therapy in adolescents. Journal of the American Academy of Child and Adolescent Psychiatry, 35, 312-318.

Quinton, D., Rutter, M. \& Liddle, C. (1984) Institutional rearing, parenting difficulties and marital support. Psychological Medicine, 14, 107-124.

Ryan, N. D. (1990) Pharmacotherapy of adolescent major depression. Beyond TCAs. Psychopharmacology Bulletin, 26, 75-79.

Simeon, J. G., Dinicola, V. F., Ferguson, H. B., et al (1990) Adolescent depression: a placebo-controlled fluoxetine treatment study and follow-up. Progress in Neuro-Psychopharmacology and Biological Psychiatry, 14, 791-795.

Vostanis, P. \& Harrington, R. C. (1994) Cognitive-behavioural treatment of depressive disorder in child psychiatric patients - rationale and description of a treatment package. European Child and Adolescent Psychiatry, 3, 111-123.

Wilkes, T. C. R., Belsher, G., Rush, A.J., et al (eds) (1994) Cognitive Therapy for Depressed Adolescents. New York: Guilford.

Wood, A., Kroll, L., Moore, A., et al (1995) Properties of the Mood and Feelings Questionnaire in adolescent psychiatric outpatients: a research note. Journal of Child Psychology and Psychiatry, 36, 327-334.

Wood, A. J., Harrington, R. C. \& Moore, A. (1996) Controlled trial of a brief cognitive-behavioural intervention in adolescent patients with depressive disorders. Journal of Child Psychology and Psychiatry, in press.

Zarb, J. M. (1992) Cognitive-Behavioral Assessment and Therapy with Adolescents. New York: Brunner/Mazel.

\section{Multiple choice questions}

1. In the diagnosis of depressive disorder in adolescence:

a High scores on depression questionnaires are usually diagnostic

b Comorbid disorders are of little consequence c Greater reliance should be placed on the history from the parents than on the interview with the child

d A family history of depression often clinches the diagnosis.

2. Common causes of resistant depression in adolescents include:

a Inadequate duration of treatment with antidepressants

b Undiagnosed bipolar disorder

c Hypothyroidism

d Undiagnosed intrafamilial stress.

3. In working with the families of depressed adolescents:

a Structural family therapy is of proven benefit

b The adolescent's symptoms can usually be understood as solving a problem for the wider family system

c The parents can be reassured that in most cases remission will occur within a few months

d The parents can be reassured that the risk of relapse is less than $15 \%$.

4. Assessment before starting an antidepressant should include:

a A depression self-report scale

b An electrocardiograph

c A full blood count

d Thyroid function tests.

5. Tricyclic antidepressants:

a Are of unproven efficacy in the treatment of major depression in adolescents

b Are contraindicated in adolescent major depression

c Are safer in adolescents than in adults

d Are associated with changes on the electrocardiograph even when the dosage is within the therapeutic range.

MCQ answers

$\begin{array}{llllllllll}\text { 1 } & & \text { 2 } & & 3 & & 4 & & 5 & \\ \text { a } & \text { F } & \text { a } & \text { T } & \text { a } & \text { F } & \text { a } & \text { T } & \text { a } & \text { T } \\ \text { b } & \text { F } & \text { b } & \text { T } & \text { b } & \text { F } & \text { b } & \text { T } & \text { b } & \text { F } \\ \text { c } & \text { F } & \text { c } & \text { F } & \text { c } & \text { T } & \text { c } & \text { T } & \text { c } & \text { F } \\ \text { d } & \text { F } & \text { d } & \text { T } & \text { d } & \text { F } & \text { d } & \text { F } & \text { d } & \text { T }\end{array}$

